\title{
Boundary layer ozone in the Northern Colorado Front Range in July-August 2014 during FRAPPE and DISCOVER-AQ from vertical profile measurements
}

\author{
S. J. Oltmans (1), L. C. Cheadle (1,2*), B. J. Johnson (1), R. C. Schnell (1), D. Helmig (3), A. M. \\ Thompson (4), P. Cullis (2,1), E. Hall (2,1), A. Jordan (2,1), C. Sterling (2,1), A. McClure- \\ Begley (2,1), J. T. Sullivan (4), T. J. McGee (4), D. Wolfe (5) \\ 1 NOAA/ESRL Global Monitoring Division, Boulder, Colorado, US \\ 2 CIRES, University of Colorado, Boulder, Colorado, US \\ 3 INSTAAR, University of Colorado, Boulder, Colorado, US \\ 4 NASA Goddard Space Flight Center, Greenbelt, Maryland, US \\ 5 NOAA/ESRL Physical Sciences Division, Boulder, Colorado, US \\ * Now at California Air Resources Board, Sacramento, California, US
}

\section{List of contents:}

Table S1: Integrated $\mathrm{O}_{3}$ in Dobson units (DU) for each tethersonde profile at Ft. Collins on August 3 and July 26, 2014 from the surface to $400 \mathrm{~m}$ agl. Also shown is the integrated column to $2000 \mathrm{~m}$ agl on August 3 based on extrapolating the top of the measured profile using a constant mixing ratio.

Table S2: Tabulation of hourly mean $\mathrm{O}_{3}$ and standard deviation for each hour of the day at the 6 $\mathrm{m}$ and $300 \mathrm{~m}$ levels at the BAO tower for July-August 2014 on days with low/moderate $\mathrm{O}_{3}(\leq 65$ ppb) and high $\mathrm{O}_{3}(\geq 75 \mathrm{ppb})$ as shown in Figure 13 .

Figure S1: Schematic of the components of the tethersonde profiling system (left) and a picture of the tethersonde in operation (right) at Ft. Collins West.

Figure S2: Comparison of surface measurements from the surface $\mathrm{O}_{3}$ monitor operated by the CDPHE and near-surface tethersonde $\mathrm{O}_{3}$ measurements with the linear regression fit at the Ft. Collins West location.

Figure S3: Ozonesondes at Platteville on July 21 and 22, 2014 with $\mathrm{O}_{3}$ (left) and temperature (right).

Figure S4: Back trajectories to Ft. Collins-West and Platteville on July 21 and 22, 2014 arriving at 1:00 PM MDT at $300 \mathrm{~m}$ agl.

Figure S5: Ozonesondes at Platteville on July 27, 2014 with $\mathrm{O}_{3}$ (left) and temperature (right).

Figure S6: Ozonesondes at Platteville on July 29, 2014 with $\mathrm{O}_{3}$ (left) and temperature (right). 
Figure S7: Back trajectories to Platteville on August 3, 2014 arriving at 1:00 PM MDT.

Figure S8: Tethersonde profiles at Ft. Collins on July 26, 2014.

Figure S9: Tethersonde profiles at Ft. Collins on August 18, 2014.

Figure S10: $\mathrm{O}_{3}$ mixing ratios at Ft. Collins West from tethersonde measurements on August 3, 2014 for $50 \mathrm{~m}$ intervals between 50-500 $\mathrm{m}$ from 8:30 AM to 5:30 PM.

Figure S11: $\mathrm{O}_{3}$ hourly mixing ratios as weekly time series at selected surface $\mathrm{O}_{3}$ monitoring sites in the CNFR during the period July 16 - August 10, 2014 covering the period of vertical profile measurements carried out during FRAPPE/DISCOVER-AQ.

Figure S12: $\mathrm{O}_{3}$ hourly average mixing ratios at two levels (6 m and $300 \mathrm{~m}$ ) (upper panel), temperature at three levels $(10 \mathrm{~m}, 100 \mathrm{~m}$ and $300 \mathrm{~m})$ (middle panel), and wind direction at three levels (lower panel) at the BAO on July 21, 2014 (upper three panels) and July 22, 2014 (lower three panels).

Figure S13: Back trajectories on days with low/moderate $\mathrm{O}_{3}$ (BAO $6 \mathrm{~m}$ peak hourly $\left.\mathrm{O} 3 \leq 65 \mathrm{ppb}\right)$ arriving at the $300 \mathrm{~m}$ tower level at 1:00 pm MDT.

Figure S14: Back trajectories on days with high $\mathrm{O}_{3}$ (BAO $6 \mathrm{~m}$ peak hourly $\mathrm{O}_{3} \geq 75 \mathrm{ppb}$ ) arriving at the $300 \mathrm{~m}$ tower level at 1:00 pm MDT.

Figure S15: $\mathrm{O}_{3}$ profiles at three times during the day (8:00 AM, 12:00 PM, and 3:00 PM on July $22 \& 23,2014$ and August $3 \&$ 18, 2014 based on measurements at surface sites along an elevation gradient. 
Table S1: Integrated $\mathrm{O}_{3}$ in Dobson units (DU) for each tethersonde profile at Ft. Collins on August 3 and July 26, 2014 from the surface to $400 \mathrm{~m}$ agl. Also shown is the integrated column to $2000 \mathrm{~m}$ agl on August 3 based on extrapolating the top of the measured profile using a constant mixing ratio.

\begin{tabular}{|c|c|c|c|c|c|c|c|c|c|c|c|c|c|}
\hline & & & & & PLOT & PLOT & & & \multirow{2}{*}{\multicolumn{4}{|c|}{$\begin{array}{c}\text { PLOT } \\
400 \text { meter }\end{array}$}} & \\
\hline Start & AGL & Mixing ratis & Column & Column & $2000 \mathrm{M}$ & $400 \mathrm{M}$ & Max Height & Max Height & & & & & Max Height \\
\hline Time & meters & Jzone ppbi & Dobson & Dobson & DU & DU & DU & meters & up and dov & & DU & & meters \\
\hline $08: 29$ & 415 & 56.4 & 1.886 & 1.821 & 8.923 & 1.376 & 1.881 & 519 & Jul 26, 2014 & $08: 00$ & 1.108 & 0.780 & 309 \\
\hline 08:34 & 414 & 56.4 & 1.932 & 1.872 & 9.299 & 1.428 & 1.927 & 518 & Jul 26, 2014 & 08:04 & 1.230 & 0.902 & 309 \\
\hline $08: 43$ & 403 & 60.2 & 1.920 & 1.909 & 9.676 & 1.452 & 1.916 & 507 & Jul 26, 2014 & $08: 13$ & & 0.228 & 95 \\
\hline 08:48 & 402 & 60.4 & 1.990 & 1.979 & 9.698 & 1.524 & 1.986 & 506 & Jul 26, 2014 & $08: 15$ & & 0.239 & 95 \\
\hline $08: 56$ & 424 & 61.5 & 2.002 & 1.893 & 9.721 & 1.420 & 1.997 & 528 & Jul 26, 2014 & $08: 39$ & 1.292 & 1.329 & 410 \\
\hline 09:01 & 424 & 61.5 & 2.013 & 1.880 & 9.745 & 1.432 & 2.005 & 528 & Jul 26, 2014 & $08: 45$ & 1.305 & 1.343 & 410 \\
\hline 09:10 & 406 & 61.4 & 1.924 & 1.897 & 9.769 & 1.416 & 1.918 & 510 & Jul 26, 2014 & $08: 57$ & 1.366 & 1.371 & 402 \\
\hline 09:15 & 406 & 61.5 & 1.988 & 1.957 & 9.932 & 1.478 & 1.982 & 510 & Jul 26, 2014 & 09:01 & 1.406 & 1.409 & 401 \\
\hline $09: 24$ & 412 & 62.3 & 2.030 & 1.979 & 10.096 & 1.491 & 2.024 & 515 & Jul 26, 2014 & 09:08 & 1.285 & 1.336 & 412 \\
\hline $09: 29$ & 411 & 62.4 & 2.082 & 2.030 & 10.097 & 1.545 & 2.076 & 515 & Jul 26, 2014 & $09: 14$ & 1.416 & 1.463 & 412 \\
\hline 09:36 & 419 & 62.0 & 2.091 & 2.002 & 10.098 & 1.524 & 2.085 & 523 & Jul 26, 2014 & $09: 20$ & 1.359 & 1.753 & 500 \\
\hline 09:41 & 418 & 62.7 & 2.125 & 2.038 & 10.164 & 1.546 & 2.122 & 522 & Jul 26, 2014 & $09: 28$ & 1.450 & 1.872 & 500 \\
\hline 10:21 & 71 & 54.1 & 0.685 & & 10.231 & 1.599 & 0.681 & 175 & Jul 26, 2014 & $09: 36$ & 1.370 & 1.372 & 401 \\
\hline $10: 22$ & 71 & 54.0 & 0.691 & & 10.273 & 1.607 & 0.689 & 175 & Jul 26, 2014 & $09: 42$ & 1.353 & 1.353 & 400 \\
\hline $10: 33$ & 409 & 62.0 & 2.180 & 2.141 & 10.316 & 1.678 & 2.173 & 513 & Jul 26, 2014 & $09: 49$ & 1.247 & 1.632 & 498 \\
\hline $10: 38$ & 408 & 62.1 & 2.246 & 2.204 & 10.356 & 1.719 & 2.239 & 512 & Jul 26, 2014 & $09: 57$ & 1.390 & 1.776 & 498 \\
\hline 10:45 & 345 & 60.5 & 1.966 & & 10.397 & 1.745 & 1.960 & 449 & Jul 26, 2014 & $10: 10$ & 1.366 & 1.366 & 400 \\
\hline $10: 52$ & 345 & 60.7 & 2.000 & & 10.380 & 1.778 & 1.994 & 449 & Jul 26, 2014 & $10: 18$ & 1.430 & 1.430 & 400 \\
\hline 11:09 & 577 & 63.5 & 3.035 & 2.229 & 10.363 & 1.761 & 3.025 & 680 & Jul 26, 2014 & $10: 28$ & 1.419 & 1.425 & 402 \\
\hline 11:17 & 577 & 63.7 & 3.149 & 2.336 & 10.447 & 1.845 & 3.135 & 680 & Jul 26, 2014 & $10: 35$ & 1.521 & 1.530 & 401 \\
\hline $11: 33$ & 404 & 64.3 & 2.396 & 2.380 & 10.531 & 1.877 & 2.388 & 508 & Jul 26, 2014 & $10: 46$ & 1.501 & 1.515 & 404 \\
\hline $11: 38$ & 404 & 63.9 & 2.434 & 2.418 & 10.615 & 1.912 & 2.425 & 508 & Jul 26, 2014 & $10: 54$ & 1.434 & 1.448 & 403 \\
\hline $12: 02$ & 500 & 64.7 & 3.052 & 2.577 & 10.700 & 2.034 & 3.042 & 604 & Jul 26, 2014 & $11: 03$ & 1.440 & 1.467 & 407 \\
\hline $12: 10$ & 499 & 64.9 & 3.108 & 2.637 & 10.922 & 2.091 & 3.096 & 603 & Jul 26, 2014 & $11: 08$ & 1.425 & 1.455 & 407 \\
\hline $12: 21$ & 405 & 71.0 & 2.674 & 2.653 & 11.144 & 2.093 & 2.666 & 509 & Jul 26, 2014 & $11: 14$ & 1.387 & 1.087 & 317 \\
\hline $12: 26$ & 404 & 71.0 & 2.641 & 2.617 & 11.169 & 2.065 & 2.630 & 508 & Jul 26, 2014 & $11: 23$ & 1.390 & 1.090 & 317 \\
\hline $12: 34$ & 406 & 71.9 & 2.703 & 2.672 & 11.194 & 2.120 & 2.695 & 510 & Jul 26, 2014 & $11: 33$ & & 0.223 & 89 \\
\hline $12: 39$ & 406 & 71.8 & 2.721 & 2.688 & 11.122 & 2.116 & 2.707 & 510 & Jul 26, 2014 & $11: 36$ & & 0.242 & 89 \\
\hline $12: 56$ & 484 & 70.4 & 3.003 & 2.578 & 11.051 & 2.039 & 2.991 & 588 & Jul 26, 2014 & $11: 37$ & 1.309 & 0.895 & 281 \\
\hline 13:02 & 484 & 70.1 & 3.038 & 2.611 & 11.213 & 2.076 & 3.025 & 588 & Jul 26, 2014 & $11: 45$ & 1.229 & 0.815 & 281 \\
\hline 13:11 & 405 & 73.1 & 2.768 & 2.742 & 11.376 & 2.171 & 2.756 & 509 & Jul 26, 2014 & $12: 02$ & 1.161 & 1.161 & 378 \\
\hline $13: 17$ & 405 & 72.7 & 2.705 & 2.677 & 11.302 & 2.114 & 2.697 & 509 & Jul 26, 2014 & $12: 09$ & 1.328 & 1.328 & 378 \\
\hline 13:44 & 401 & 71.3 & 2.700 & 2.696 & 11.229 & 2.136 & 2.692 & 505 & Jul 26, 2014 & $12: 20$ & 1.376 & 1.393 & 406 \\
\hline $13: 49$ & 401 & 71.5 & 2.617 & 2.614 & 11.303 & 2.058 & 2.611 & 505 & Jul 26, 2014 & $12: 25$ & 1.464 & 1.482 & 406 \\
\hline 13:57 & 412 & 73.1 & 2.797 & 2.730 & 11.376 & 2.164 & 2.789 & 516 & Jul 26, 2014 & $12: 36$ & 1.449 & 1.035 & 299 \\
\hline $14: 02$ & 412 & 73.1 & 2.800 & 2.740 & 11.349 & & & & Jul 26, 2014 & $12: 43$ & 1.395 & 0.981 & 298 \\
\hline $14: 13$ & 399 & 72.0 & 2.719 & 2.719 & 11.322 & 2.167 & 2.711 & 503 & Jul 26, 2014 & $13: 25$ & 1.655 & 1.470 & 346 \\
\hline $14: 18$ & 399 & 72.0 & 2.801 & 2.801 & 11.728 & 2.243 & 2.792 & 502 & Jul 26, 2014 & $13: 30$ & 1.634 & 1.449 & 346 \\
\hline $14: 31$ & 404 & 85.0 & 3.090 & 3.064 & 12.134 & 2.415 & 3.079 & 508 & & & & & \\
\hline $14: 37$ & 404 & 84.1 & 3.100 & 3.074 & 12.041 & 2.432 & 3.090 & 508 & & & & & \\
\hline $14: 50$ & 447 & 79.8 & 3.151 & 2.884 & 11.948 & 2.282 & 3.139 & 551 & & & & & \\
\hline $14: 57$ & 447 & 79.7 & 3.294 & 3.028 & 12.365 & 2.401 & 3.279 & 551 & & & & & \\
\hline 15:07 & 402 & 86.4 & 3.162 & 3.154 & 12.782 & 2.475 & 3.150 & 506 & & & & & \\
\hline $15: 13$ & 401 & 86.8 & 3.225 & 3.220 & 12.907 & 2.539 & 3.205 & 505 & & & & & \\
\hline $15: 24$ & 441 & 87.3 & 3.476 & 3.214 & 13.033 & 2.542 & 3.463 & 545 & & & & & \\
\hline $15: 34$ & 440 & 87.2 & 3.351 & 3.094 & 12.706 & 2.446 & 3.345 & 544 & & & & & \\
\hline $16: 30$ & 403 & 82.4 & 3.092 & 3.074 & 12.379 & 2.437 & 3.083 & 507 & & & & & \\
\hline $16: 35$ & 403 & 82.8 & 3.078 & 3.057 & 12.456 & 2.420 & 3.069 & 507 & & & & & \\
\hline $16: 42$ & 505 & 85.9 & 3.784 & 3.152 & 12.532 & 2.487 & 3.774 & 609 & & & & & \\
\hline $16: 50$ & 505 & 86.9 & 3.601 & 2.974 & 12.321 & 2.354 & 3.582 & 609 & & & & & \\
\hline $16: 59$ & 392 & 79.9 & 3.009 & 3.009 & 12.110 & 2.439 & 2.998 & 496 & & & & & \\
\hline $17: 07$ & 391 & 82.2 & 2.937 & 2.940 & 12.092 & 2.357 & 2.916 & 495 & & & & & \\
\hline $17: 18$ & 208 & 82.2 & 1.890 & & 12.073 & 2.421 & 1.881 & 312 & & & & & \\
\hline $17: 22$ & 208 & 80.9 & 1.873 & & 12.050 & & & & & & & & \\
\hline
\end{tabular}


Table S2: Tabulation of hourly mean $\mathrm{O}_{3}$ and standard deviation for each hour of the day at the 6 $\mathrm{m}$ and $300 \mathrm{~m}$ levels at the BAO tower for July-August 2014 on days with low/moderate $\mathrm{O}_{3}(\leq 65$ $\mathrm{ppb})$ and high $\mathrm{O}_{3}(\geq 75 \mathrm{ppb})$ as shown in Figure 13.

\begin{tabular}{|c|c|c|c|c|c|c|c|c|}
\hline \multirow{3}{*}{$\begin{array}{c}\text { Hour } \\
\text { of } \\
\text { Day }\end{array}$} & \multicolumn{4}{|c|}{$6 \mathrm{~m} \mathrm{Peak} \mathrm{O}_{3} \leq 65 \mathrm{ppb}$} & \multicolumn{4}{|c|}{$6 \mathrm{~m} \mathrm{Peak} \mathrm{O}_{3} \geq 75 \mathrm{ppb}$} \\
\hline & \multicolumn{2}{|c|}{$6 \mathrm{~m} \mathrm{O}_{3}(\mathrm{ppb})$} & \multicolumn{2}{|c|}{$300 \mathrm{~m} \mathrm{O}_{3}(\mathrm{ppb})$} & \multicolumn{2}{|c|}{$6 \mathrm{~m} \mathrm{O}_{3}(\mathrm{ppb})$} & \multicolumn{2}{|c|}{$300 \mathrm{~m} \mathrm{O}_{3}(\mathrm{ppb})$} \\
\hline & Mean & $\begin{array}{l}\text { Standard } \\
\text { Deviation }\end{array}$ & Mean & $\begin{array}{l}\text { Standard } \\
\text { Deviation }\end{array}$ & Mean & $\begin{array}{l}\text { Standard } \\
\text { Deviation }\end{array}$ & Mean & $\begin{array}{l}\text { Standard } \\
\text { Deviation }\end{array}$ \\
\hline 1 & 28.4173 & 7.8225 & 53.0204 & 7.5767 & 29.7033 & 6.1519 & 54.5322 & 9.9779 \\
\hline 2 & 26.1631 & 7.9096 & 53.1302 & 8.9906 & 28.5767 & 8.0591 & 52.1889 & 9.1572 \\
\hline 3 & 26.1536 & 8.5503 & 52.1920 & 8.1748 & 27.2100 & 8.0502 & 47.8722 & 10.6510 \\
\hline 4 & 26.4298 & 8.8826 & 50.2902 & 7.3390 & 24.5589 & 4.7880 & 47.5500 & 9.6186 \\
\hline 5 & 24.9627 & 8.8385 & 50.0838 & 7.6449 & 23.5789 & 6.5622 & 50.5378 & 9.7604 \\
\hline 6 & 23.5069 & 9.2183 & 48.2918 & 7.6208 & 20.8822 & 4.2248 & 48.9289 & 7.4604 \\
\hline 7 & 22.1407 & 7.6414 & 47.6602 & 8.7853 & 26.4133 & 8.0246 & 48.5656 & 9.0228 \\
\hline 8 & 24.7480 & 7.1876 & 47.9340 & 8.3818 & 31.9775 & 7.1209 & 51.3588 & 9.3752 \\
\hline 9 & 29.1562 & 9.3520 & 48.6462 & 7.4301 & 37.2850 & 7.7861 & 49.8575 & 8.9548 \\
\hline 10 & 34.5669 & 9.2613 & 48.2627 & 8.1738 & 42.0611 & 8.5723 & 48.4944 & 8.9765 \\
\hline 11 & 41.8882 & 9.2717 & 49.5709 & 7.0333 & 48.6789 & 9.3287 & 52.7656 & 8.7084 \\
\hline 12 & 48.4582 & 7.9782 & 54.6836 & 8.6814 & 58.0567 & 7.8638 & 59.4189 & 9.3338 \\
\hline 13 & 52.4484 & 7.1370 & 59.7109 & 8.7176 & 66.6711 & 9.4432 & 70.8056 & 9.9362 \\
\hline 14 & 52.9331 & 8.2388 & 62.3927 & 7.9382 & 72.7400 & 6.9033 & 79.6622 & 12.5690 \\
\hline 15 & 53.1036 & 7.8535 & 62.9733 & 7.3543 & 70.6711 & 7.4337 & 81.8444 & 7.9607 \\
\hline 16 & 51.9289 & 8.3260 & 62.6549 & 7.1796 & 64.0856 & 10.0442 & 76.3111 & 9.0104 \\
\hline 17 & 50.6871 & 8.3561 & 61.9582 & 7.8893 & 60.7389 & 9.8153 & 70.7422 & 9.0801 \\
\hline 18 & 49.3655 & 7.9518 & 60.5489 & 7.2515 & 55.6133 & 13.0534 & 68.9122 & 10.5285 \\
\hline 19 & 46.0516 & 8.9882 & 59.8186 & 7.4980 & 52.7756 & 14.6572 & 67.6667 & 13.3900 \\
\hline 20 & 41.2845 & 8.7015 & 58.2198 & 7.0888 & 47.8433 & 9.7819 & 65.2778 & 13.8522 \\
\hline 21 & 37.1243 & 8.7825 & 57.8132 & 7.7793 & 38.5889 & 5.7202 & 62.9267 & 12.0115 \\
\hline 22 & 33.7680 & 7.8436 & 55.7457 & 8.5759 & 33.0122 & 4.6905 & 61.9733 & 12.1655 \\
\hline 23 & 31.1148 & 8.1503 & 55.2364 & 7.5727 & 31.3144 & 5.0058 & 54.9956 & 9.3750 \\
\hline 24 & 28.2975 & 6.9745 & 53.5098 & 7.6810 & 33.7700 & 6.1793 & 53.9411 & 6.8279 \\
\hline
\end{tabular}




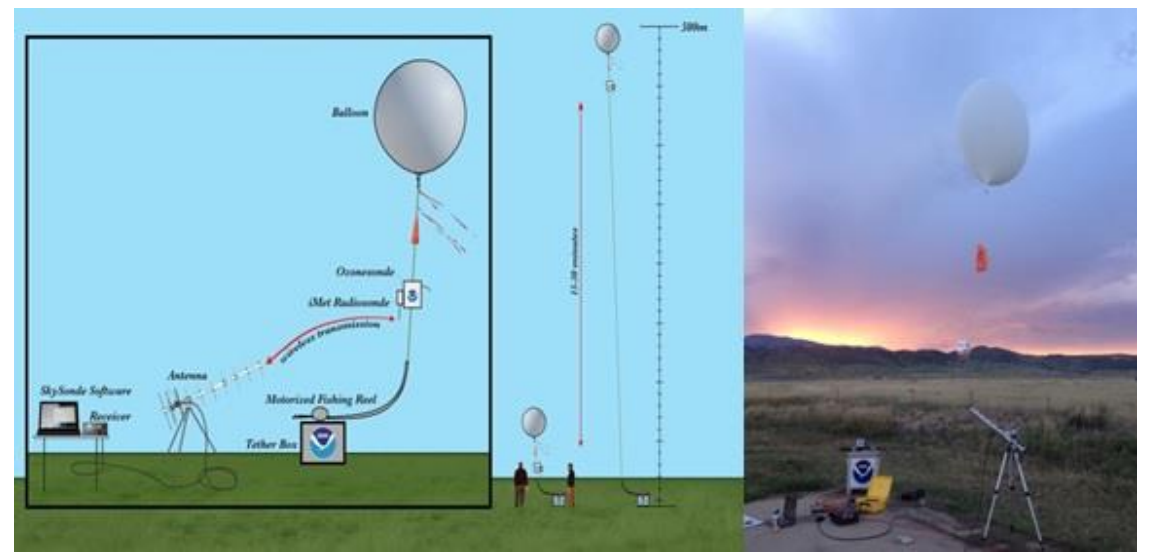

Figure S1: Schematic of the components of the tethersonde profiling system (left) and a picture of the tethersonde in operation (right) at Ft. Collins West.

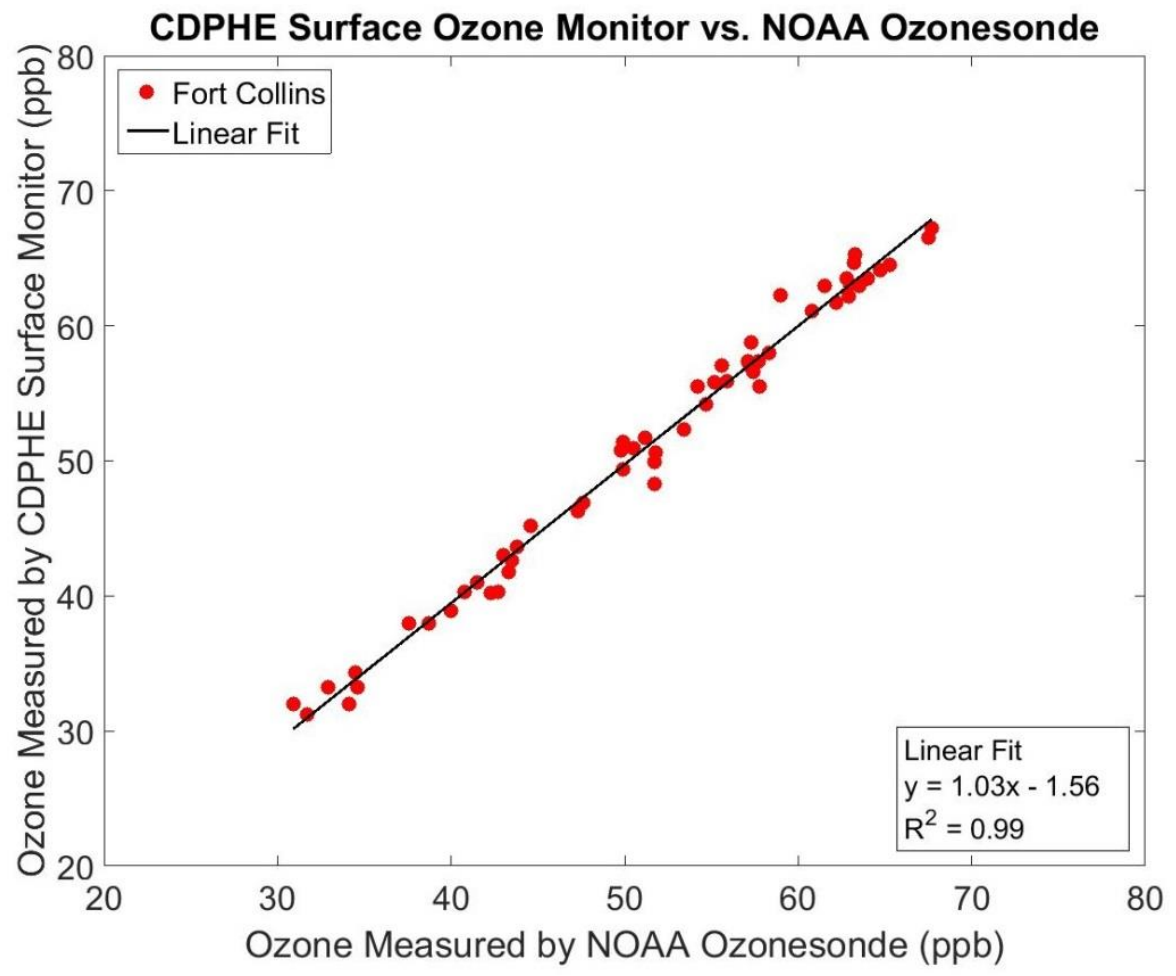

Figure S2: Comparison of surface measurements from the surface $\mathrm{O}_{3}$ monitor operated by the CDPHE and near-surface tethersonde $\mathrm{O}_{3}$ measurements with the linear regression fit at the Ft. Collins West location. 

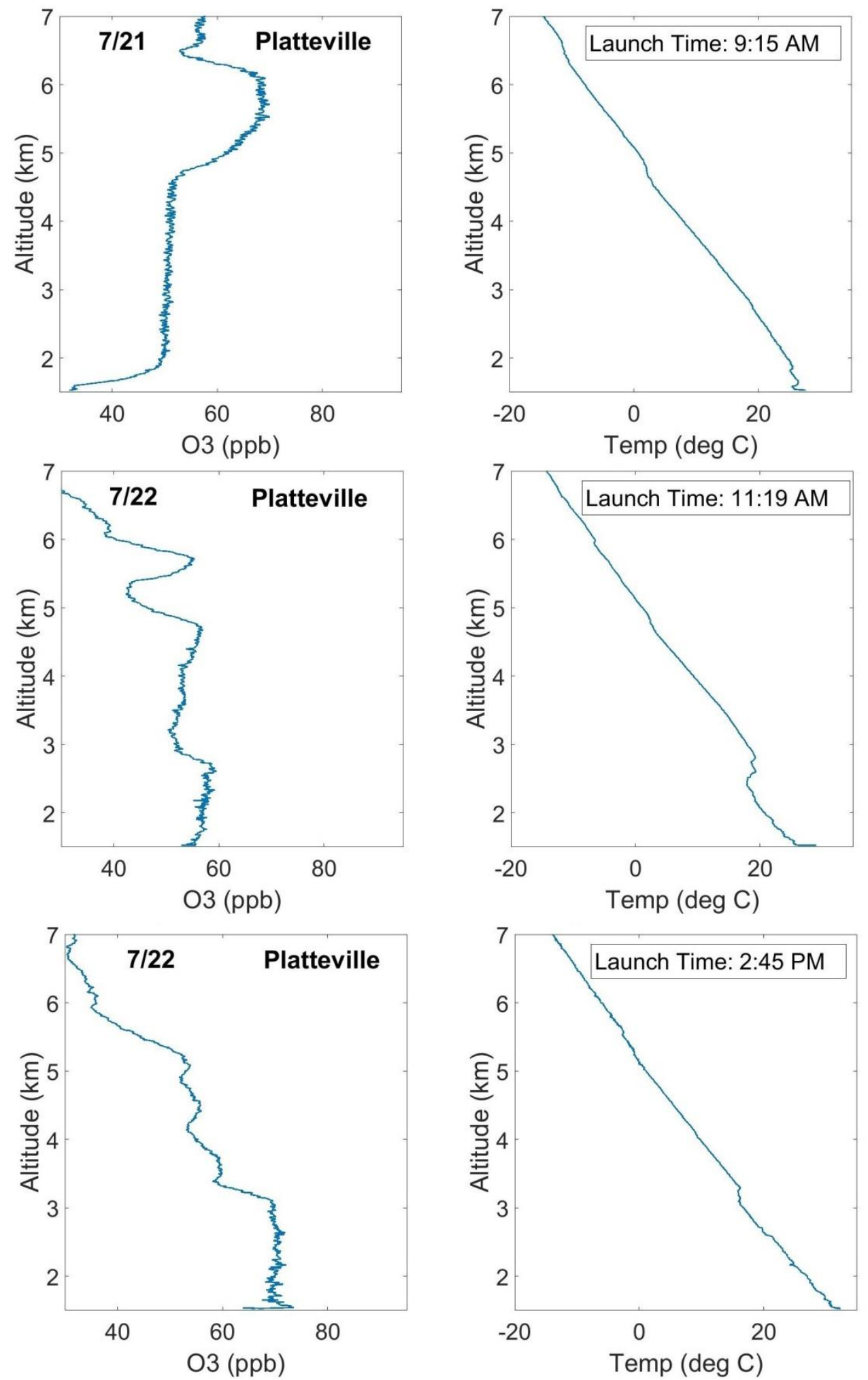

Figure S3: Ozonesondes at Platteville on July 21 and 22, 2014 with $\mathrm{O}_{3}$ (left) and temperature (right). 

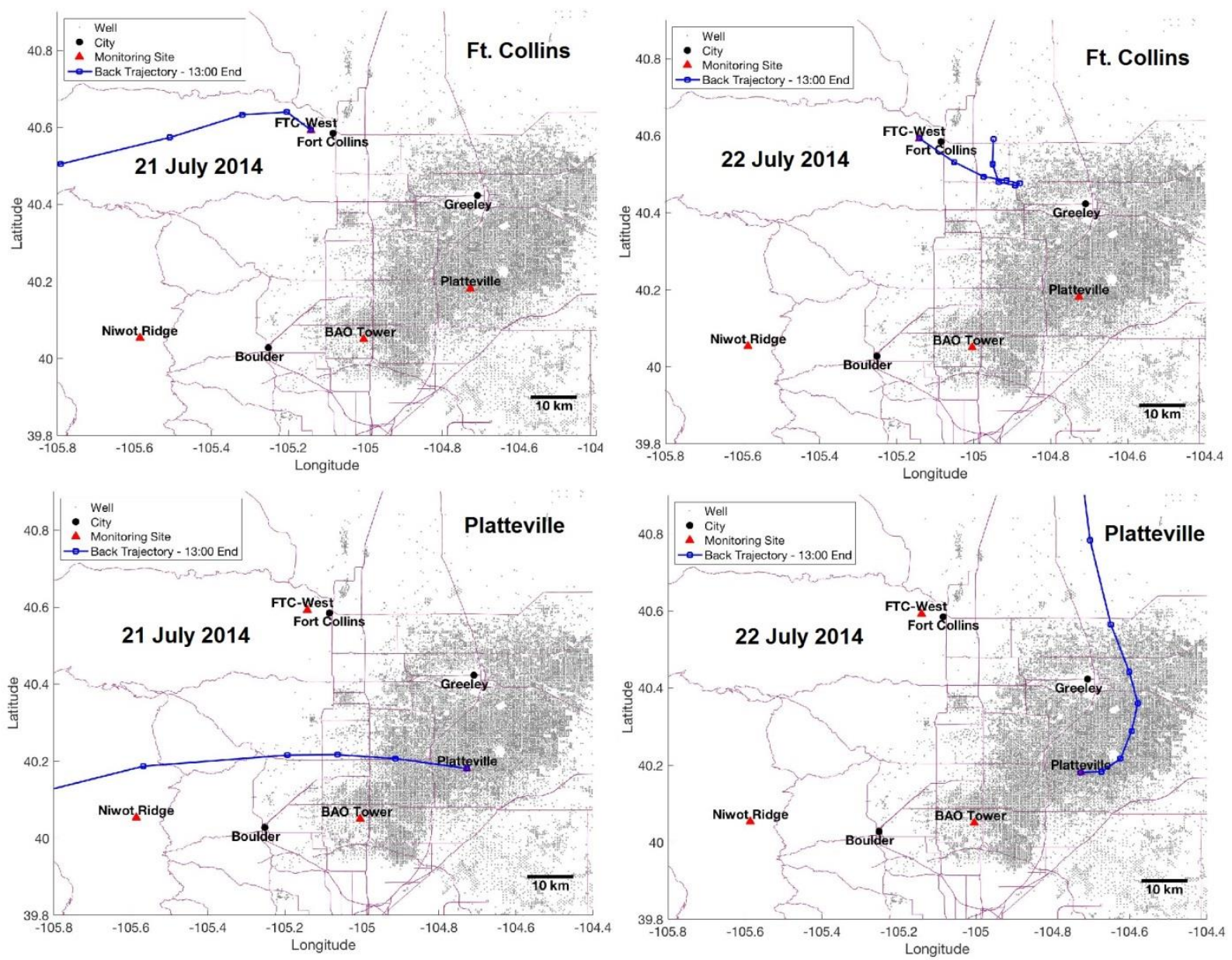

Figure S4: Back trajectories to Ft. Collins-West and Platteville on July 21 and 22, 2014 arriving at 1:00 PM MDT. 

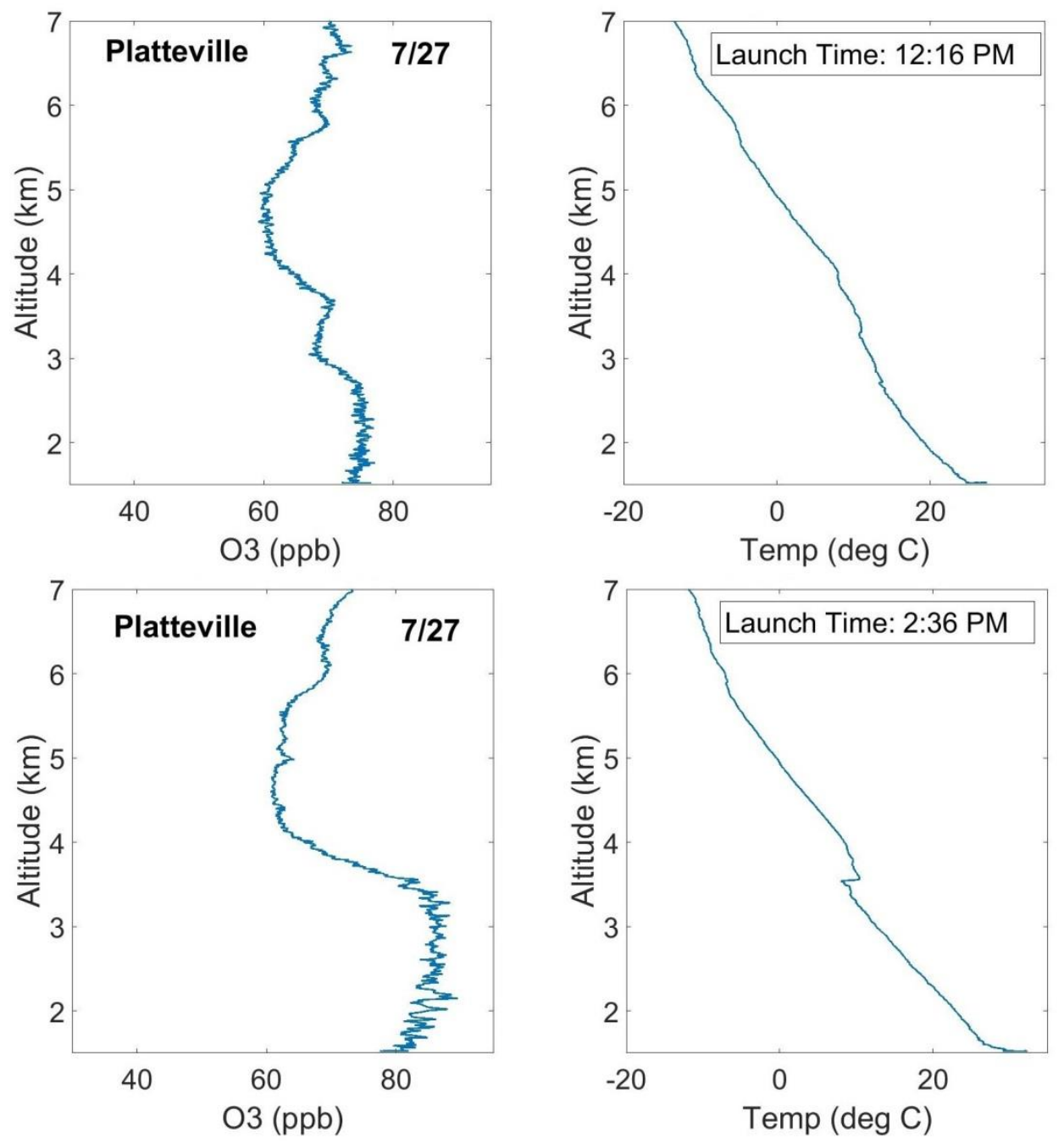

Figure S5: Ozonesondes at Platteville on July 27, 2014 with $\mathrm{O}_{3}$ (left) and temperature (right). 

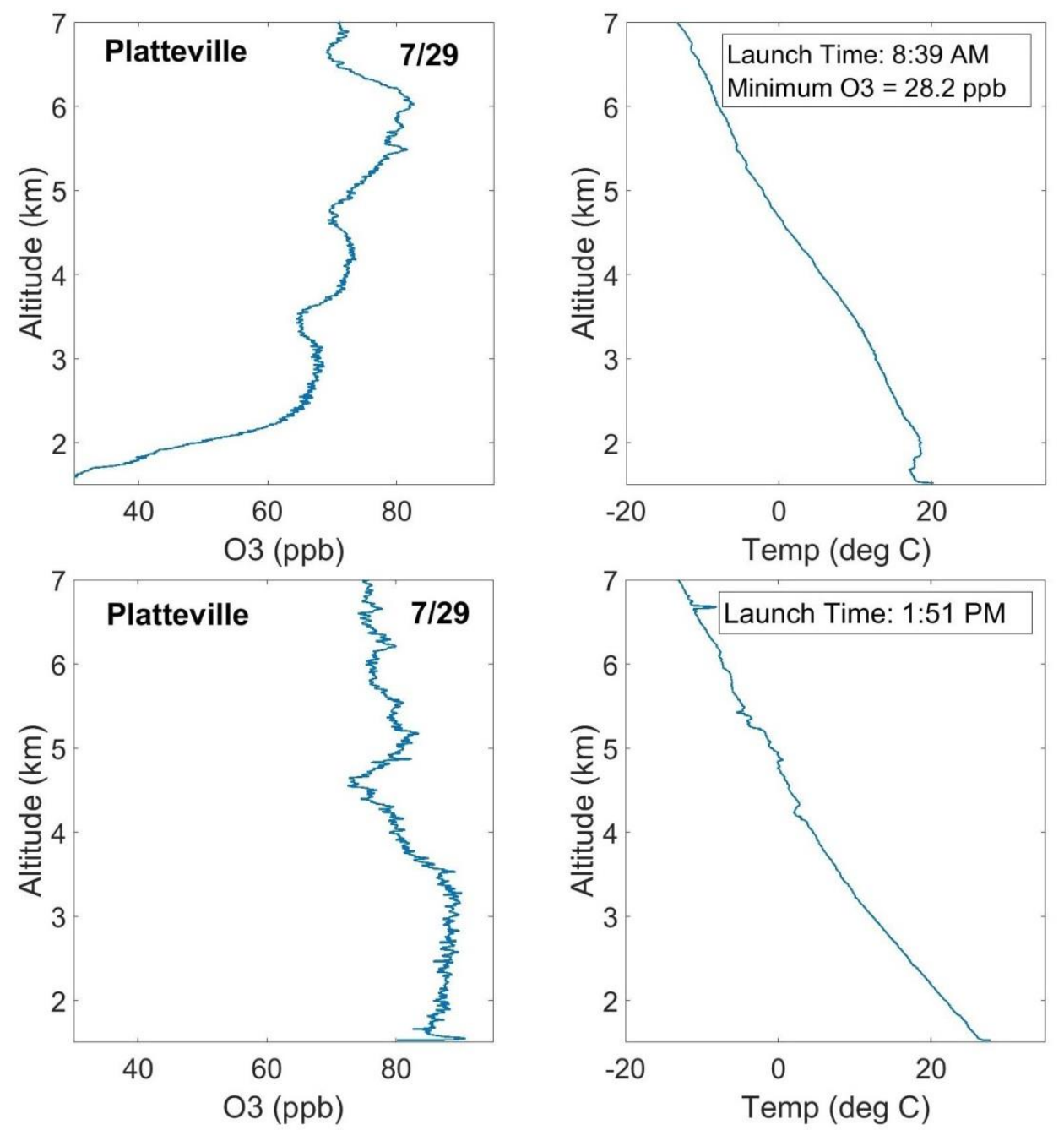

Figure S6: Ozonesondes at Platteville on July 29, 2014 with $\mathrm{O}_{3}$ (left) and temperature (right). 

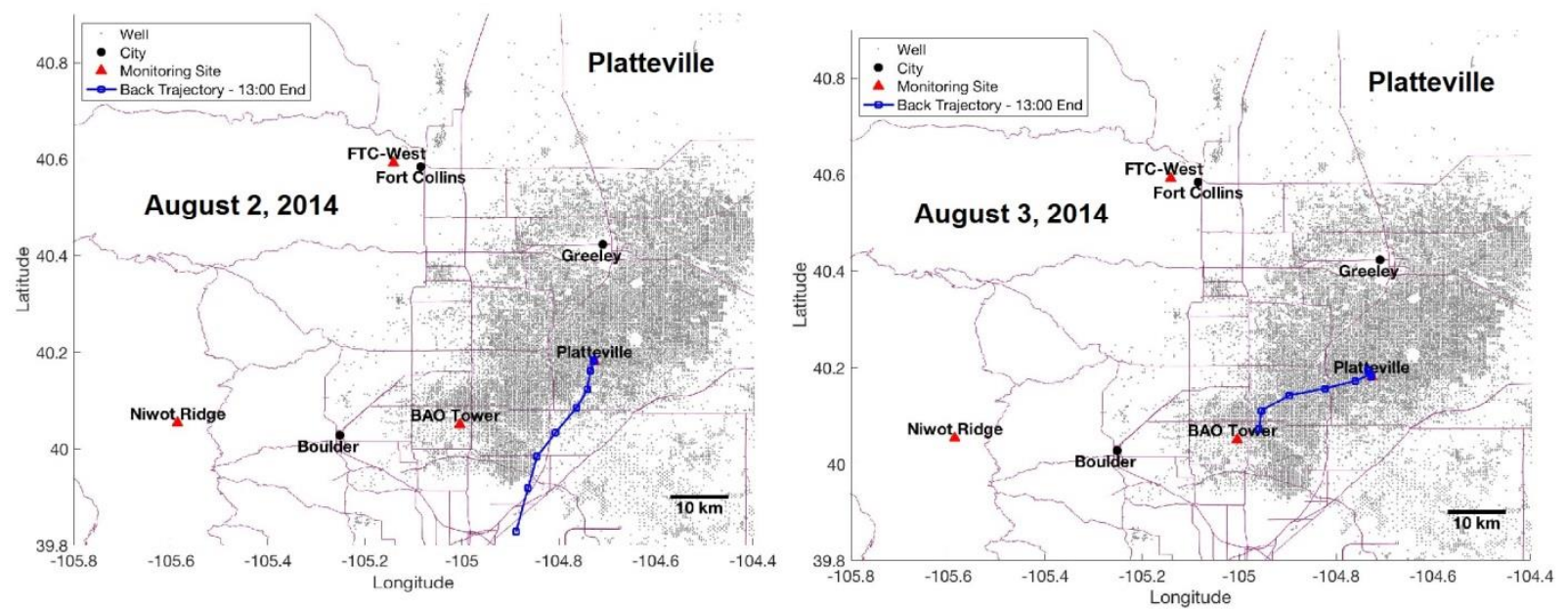

Figure S7: Back trajectories to Platteville on August 3, 2014 arriving at 1:00 PM MDT. 


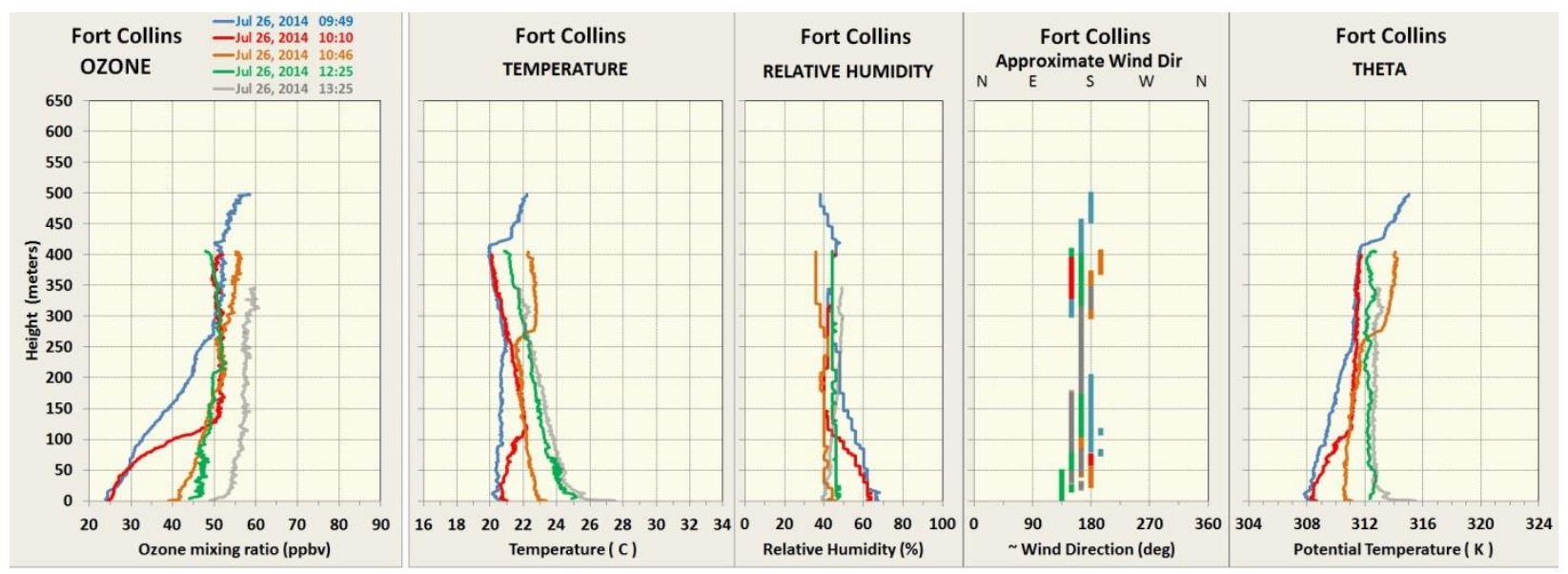

Figure S8: Tethersonde profiles at Ft. Collins on July 26, 2014.

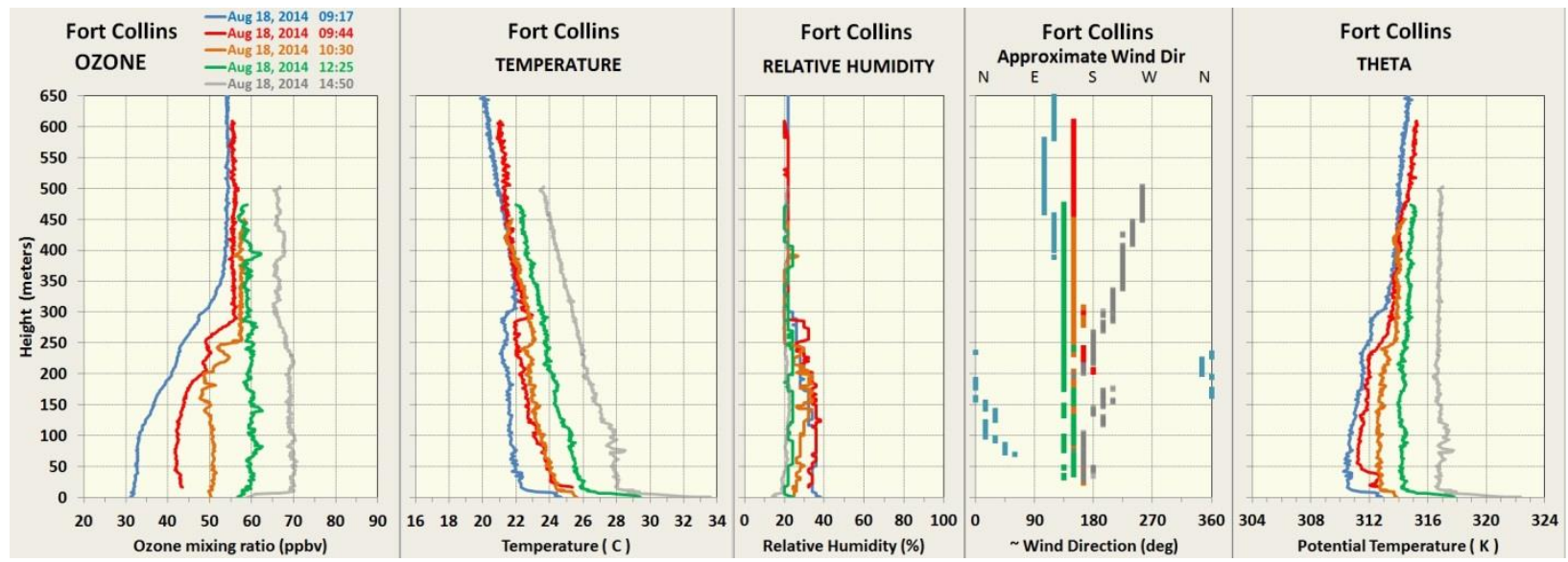

Figure S9: Tethersonde profiles at Ft. Collins on August 18, 2014. 


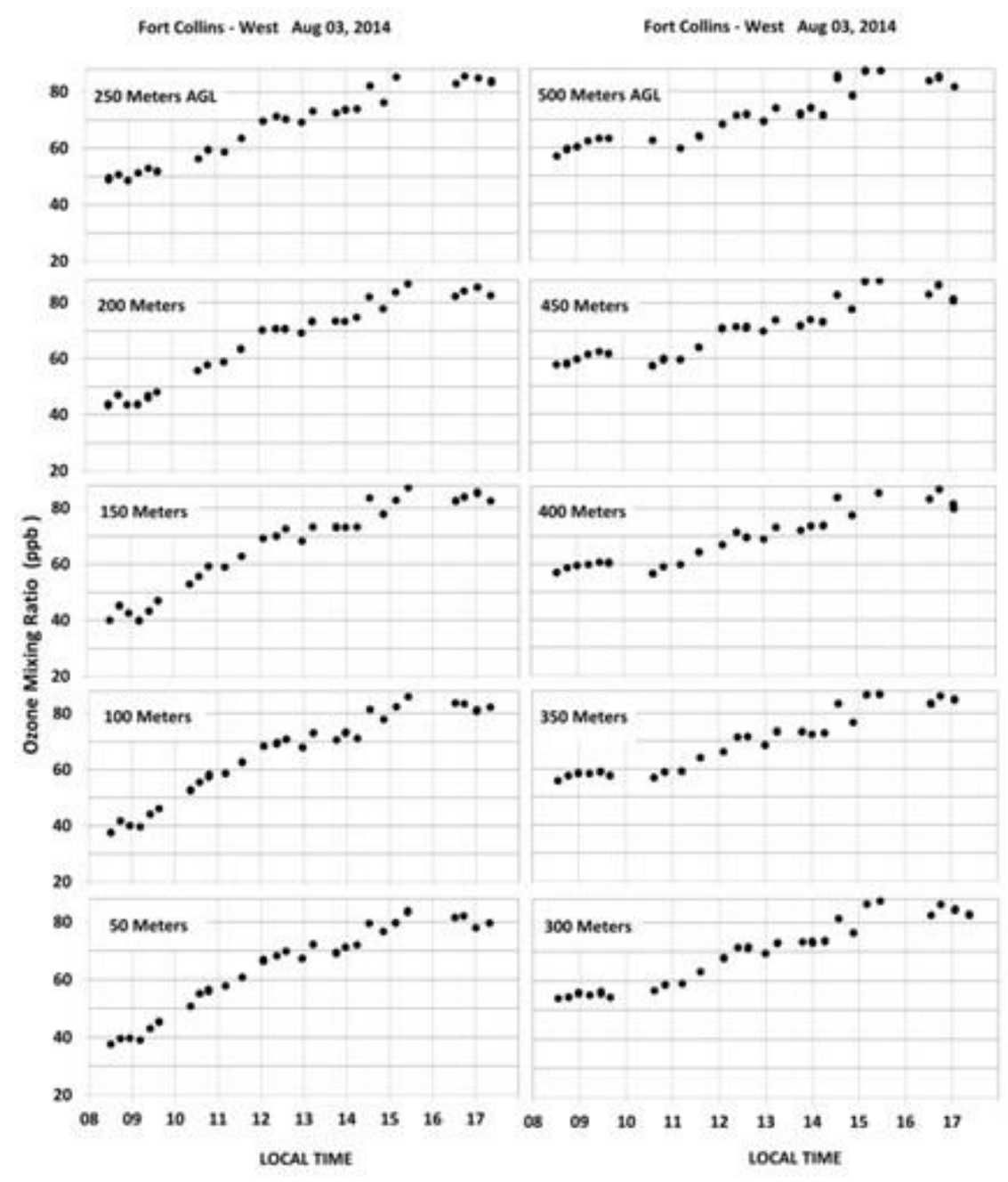

Figure S10: $\mathrm{O}_{3}$ mixing ratios at Ft. Collins West from tethersonde measurements on August 3, 2014 for 50 m intervals between 50-500 m from 8:30 AM to 5:30 PM. 

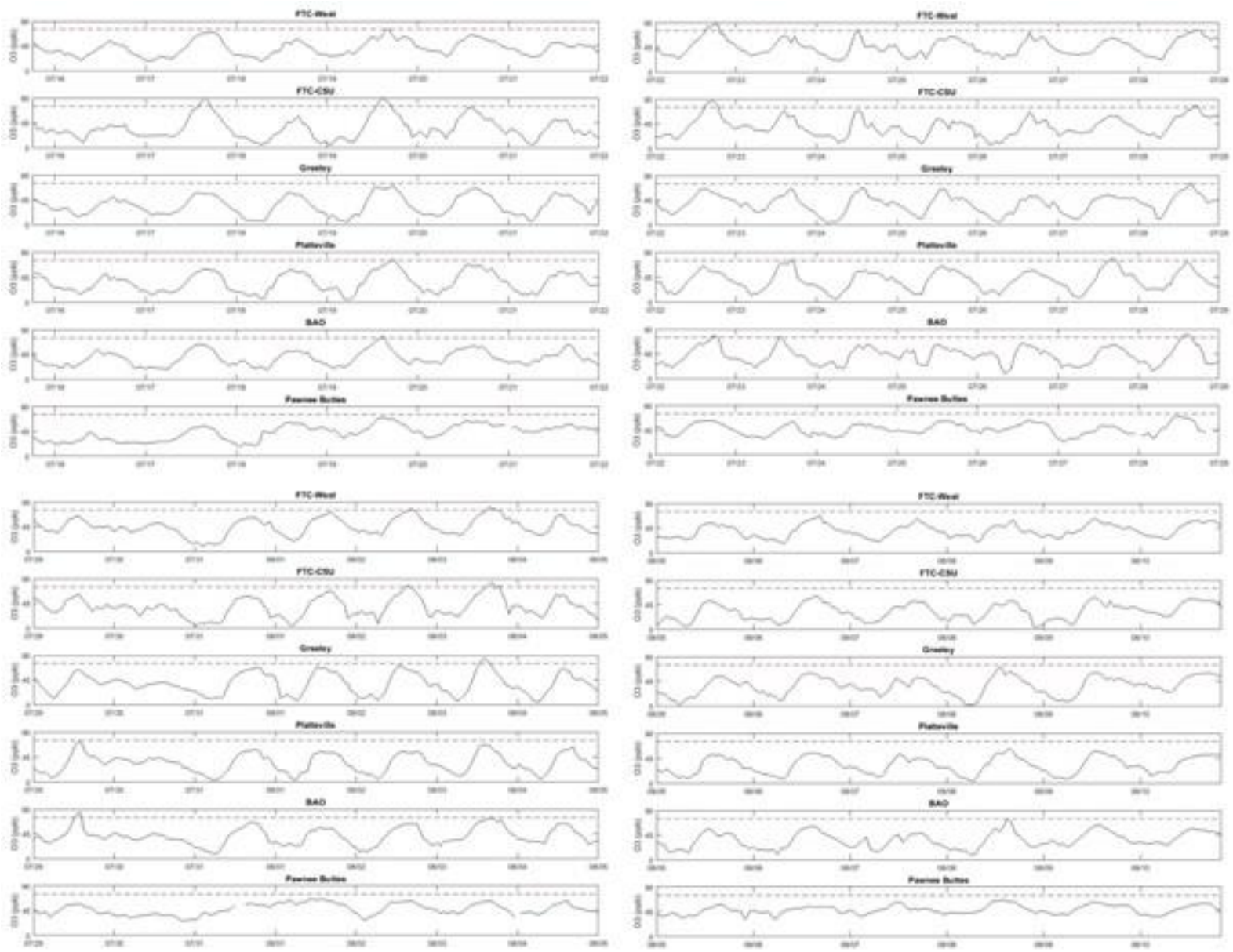

Figure S11: $\mathrm{O}_{3}$ hourly mixing ratios as weekly time series at selected surface $\mathrm{O}_{3}$ monitoring sites in the CNFR during the period July 16 - August 10, 2014 covering the period of vertical profile measurements carried out during FRAPPE/DISCOVER-AQ. 

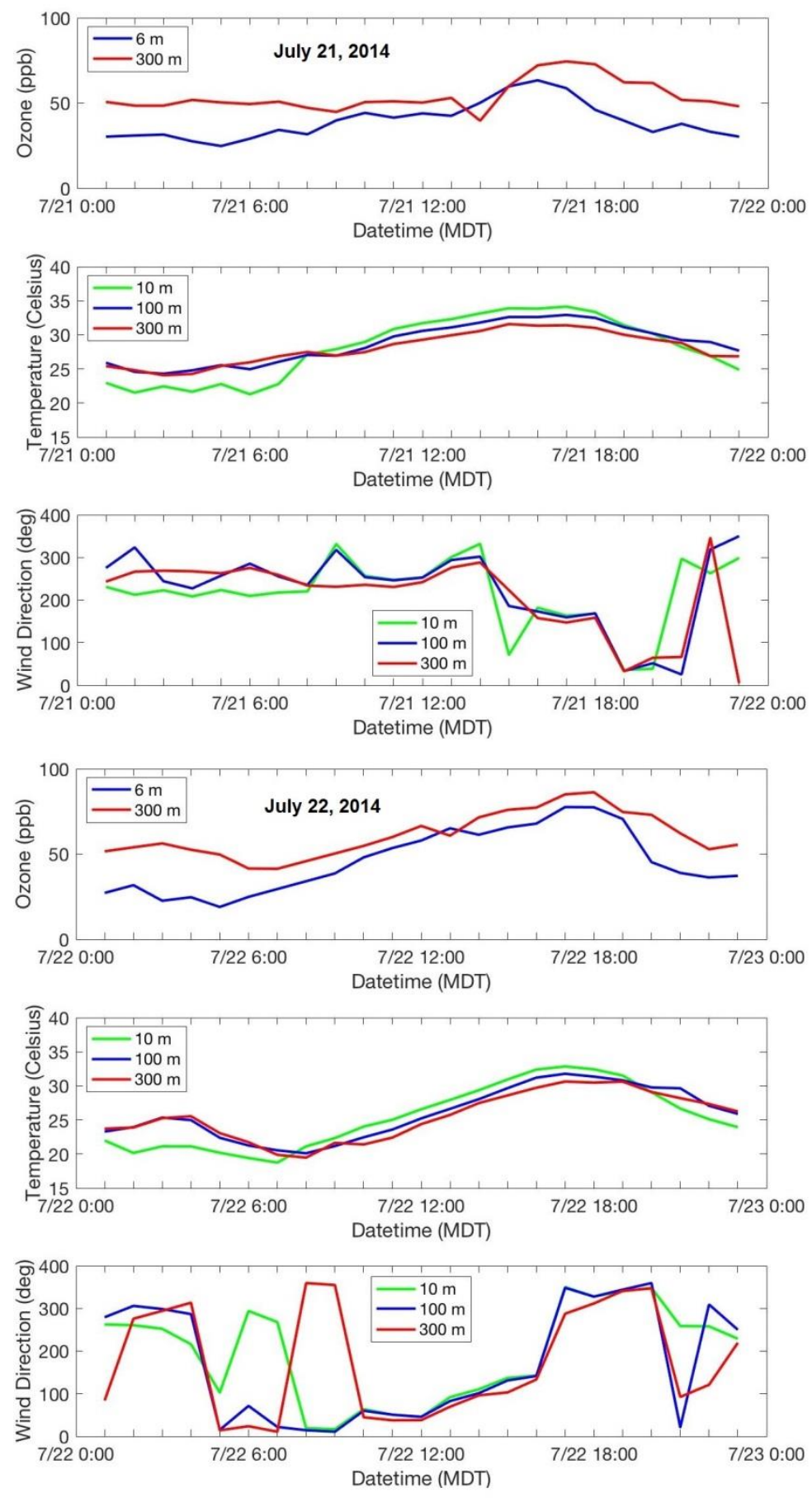

Figure S12: $\mathrm{O}_{3}$ hourly average mixing ratios at two levels (6 $\mathrm{m}$ and $300 \mathrm{~m}$ ) (upper panel), temperature at three levels (10 m, $100 \mathrm{~m}$ and $300 \mathrm{~m}$ ) (middle panel), and wind direction at three levels (lower panel) at the BAO on July 21, 2014 (upper three panels) and July 22, 2014 (lower three panels). 

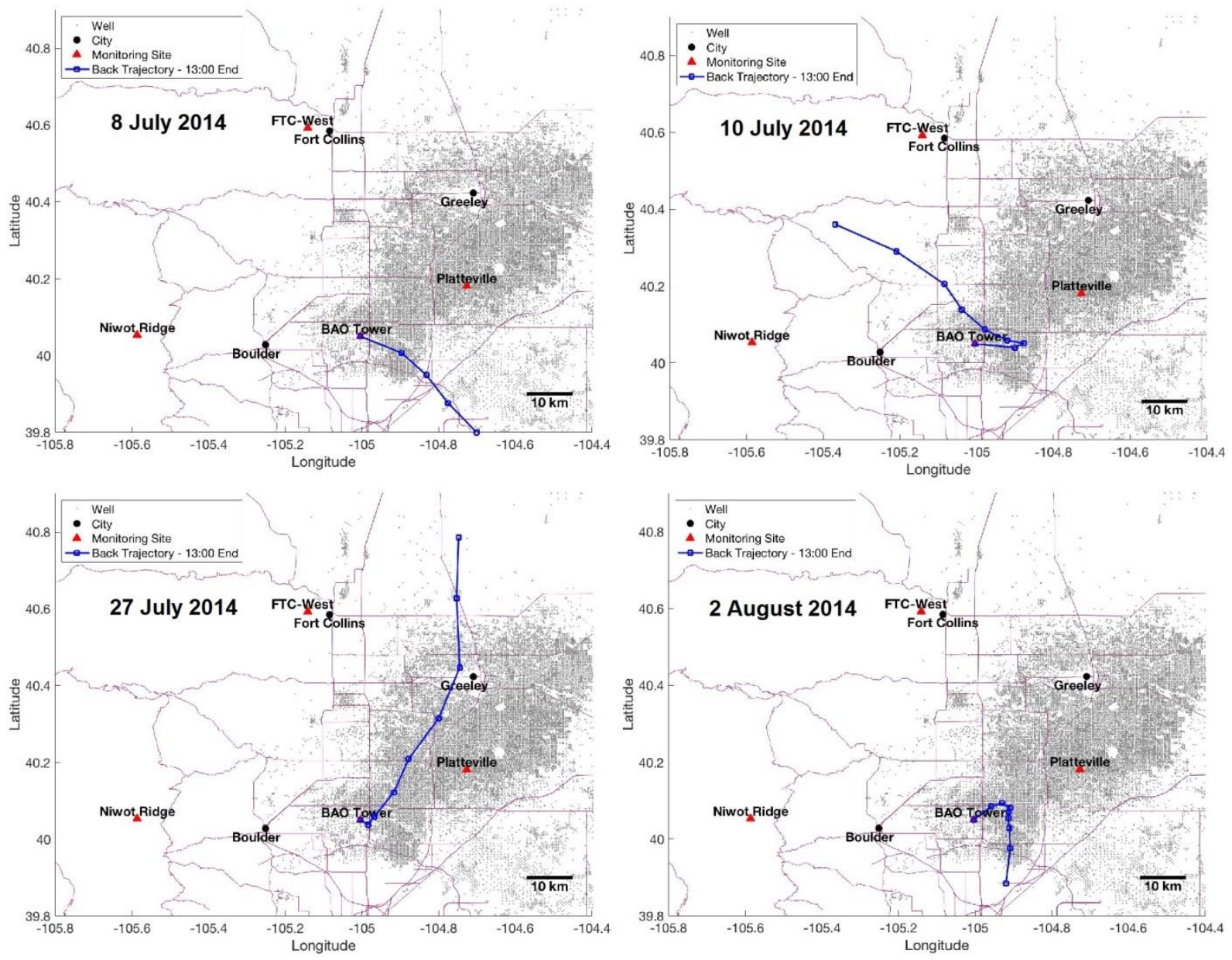

Figure S13: Back trajectories on days with low/moderate $\mathrm{O}_{3}\left(\mathrm{BAO} 6 \mathrm{~m}\right.$ peak hourly $\left.\mathrm{O}_{3} \leq 65 \mathrm{ppb}\right)$ arriving at the $300 \mathrm{~m}$ tower level at 1:00 pm MDT. 

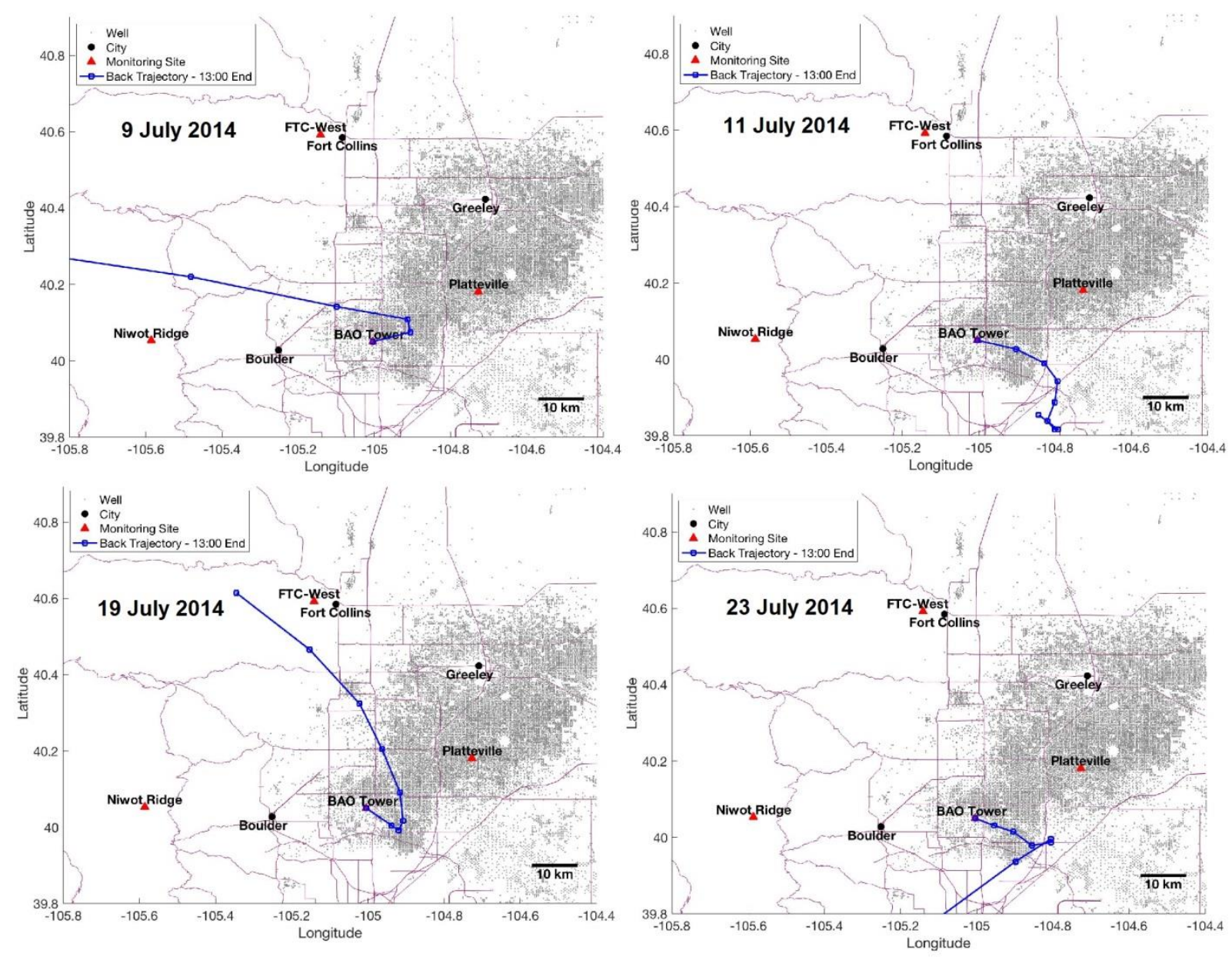

Figure S14: Back trajectories on days with high $\mathrm{O}_{3}\left(\mathrm{BAO}\right.$ 6m peak hourly $\left.\mathrm{O}_{3} \geq 75 \mathrm{ppb}\right)$ arriving at the $300 \mathrm{~m}$ tower level at 1:00 pm MDT. 

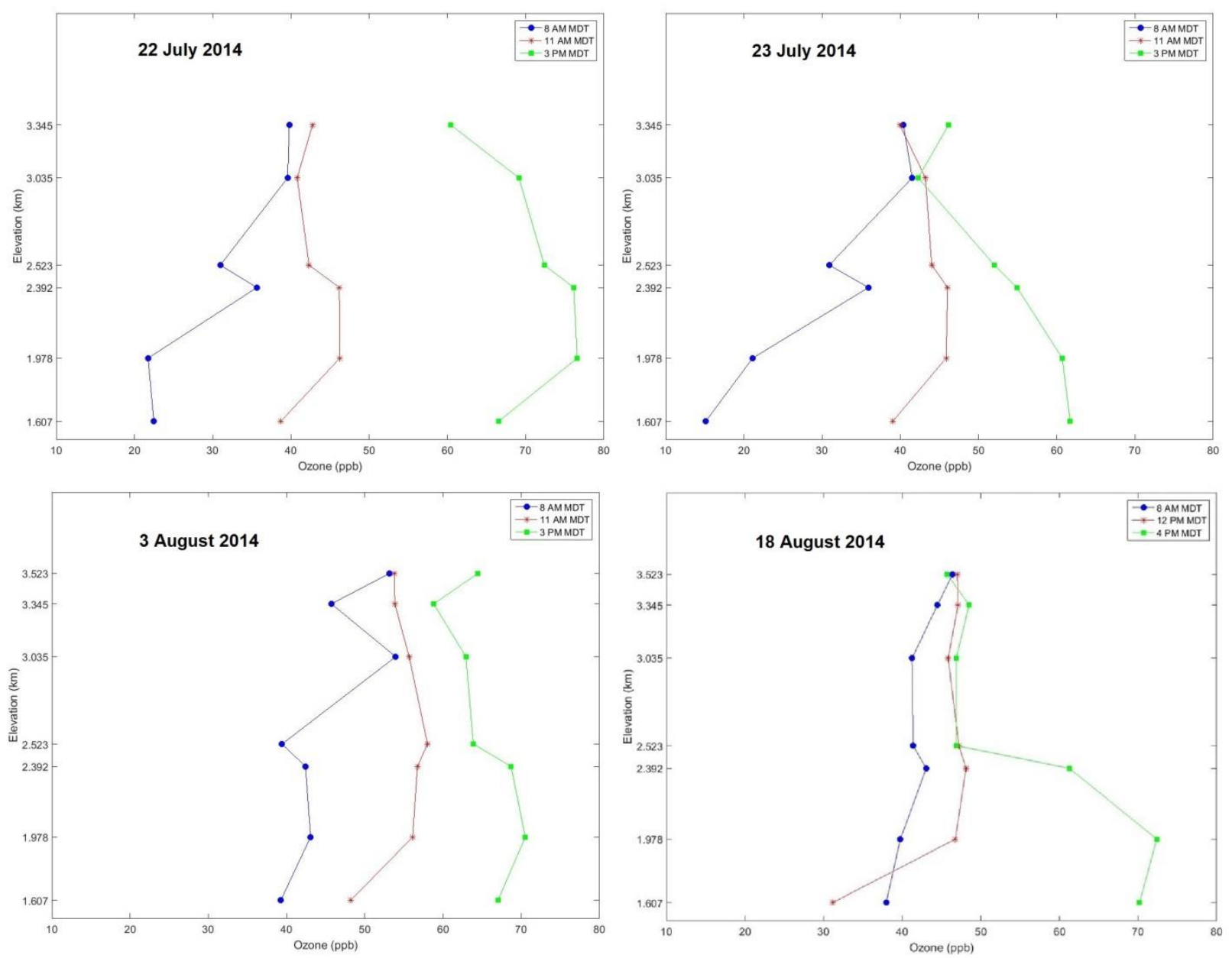

Figure S15: $\mathrm{O}_{3}$ profiles at three times during the day (8:00 AM, 12:00 PM, and 3:00 PM on July 22 and 23, 2014 and August 3 and 18, 2014 based on measurements at surface sites along an elevation gradient. 